\title{
Diseño y construcción de un cañón de gas de una etapa para pruebas de impacto de alta velocidad
}

\section{Design and Construction of a One-Stage Gas Gun for High Velocity Impact Tests}

\author{
Gamboa-Castellanos Ricardo Alberto \\ Centro de Investigación Científica de Yucatán AC \\ Unidad de Materiales, Mérida \\ Correo: ragcicy@cicy.mx \\ Carrillo-Baeza José Gonzalo \\ Centro de Investigación Científica de Yucatán AC \\ Unidad de Materiales, Mérida \\ Correo:jgcb@cicy.mx
}

\author{
Flores-Johnson Emmanuel Alejandro \\ The University of Sydney \\ School of Civil Engineering, Sydney, Australia \\ Correo:emmanuel.flores-johnson@sydney.edu.au
}

Información del artículo: recibido: septiembre de 2013, reevaluado: febrero de 2014, aceptado: abril de 2014

\section{Resumen}

Las pruebas de alto impacto se caracterizan por las altas velocidades involucradas, así como la complejidad de los acontecimientos debido a la rapidez con la que estos ocurren; sin embargo, recurrir a estas pruebas se ha vuelto cada vez más popular, debido a los requerimientos de seguridad necesarios en estos días. En el presente documento se diseña y construye un cañón de gas ligero de una etapa para pruebas de alto impacto; este diseño en particular se caracteriza por su simplicidad, relación precio-rendimiento y dimensiones, en comparación con sistemas comerciales, presentando una alternativa accesible para la caracterización de materiales ante pruebas de impacto a alta velocidad. Los resultados se plasman en la caracterización de un blindaje termoplástico, donde se obtiene el límite balístico del material, al mismo tiempo que se comprueba la efectividad y versatilidad del equipo.
\end{abstract}

\footnotetext{
Abstract

High impact tests are characterized by a projectile traveling at high speed as well as complex events such as flying fragments due to the velocity at which the impact occurs; however, these tests have become increasingly popular due to need for more stringent protective material requirements nowadays. In this paper, the design and construction of a one-stage light gas gun for ballistic testing is presented. This particular design is characterized by its simplicity, excellent performance at low cost and compact dimensions, when compared to commercial systems, presenting an affordable option for materials characterization for high velocity impact tests. The results are completed with the characterization of an armor grade material, obtaining the ballistic limit of the material, as well as demonstrating the effectiveness and versatility of the equipment.
}

\section{Descriptores:}

- diseño de equipo

- equipo de laboratorio

- impactos de alta velocidad

- prueba dinámica de impacto

- caracterización mecánica
Keywords:
- equipment design
- laboratory equipment
- high velocity impact
- impact dynamic test
- mechanical characterization 


\section{Introducción}

Las pruebas de impacto a altas velocidades se caracterizan por la cantidad de parámetros que pueden involucrar para predecir el comportamiento de un material ante un proyectil determinado; entre los cuales se puede mencionar la geometría y el material del proyectil, el ángulo de impacto en el blanco, la forma de sujeción del material, distancia del impacto, entre otros (Donald et al., 2008). Debido a la naturaleza estocástica que estos parámetros generan en una prueba de impacto, surgió la necesidad de desarrollar estándares de prueba, entre los que se puede mencionar la Military Standard MIL - 662F, NIJ Estándar 0101.04, PSDB Ballistic Body Armor Standard, STANAG 2920, RENAR M.01 y la Norma Oficial Mexicana NOM-142-SCFI-2000. Todas estas normas se caracterizan por utilizar un tipo de equipo en particular para realizar las pruebas; un cañón de gas para pruebas de impacto (Ashok, 2006; Registro Nacional de Armas, 2001; Direccion Nacional de Normas 2001).

Un cañón de gas se basa en la generación de alta presión en un recipiente cerrado, la cual es liberada repentinamente, canalizando la energía cinética del gas contenido para acelerar un proyectil, que se impactará contra un blanco (Seigel, 1965). De este funcionamiento básico se han generado varios diseños, los cuales se enfocan a distintas áreas de investigación; como el Shock tube, el cual se utiliza para hacer impactar trozos pequeños de material en un sustrato y para poner a prueba su resistencia ante ondas de expansión en situaciones con explosivos. Otro de los equipos ampliamente utilizado es el cañón de gas ligero de dos etapas (TSLGG).

El TSLGG consiste en dos cañones de diferente tamaño, el primero llamado cámara de bombeo (pump tube) es donde ocurre una explosión controlada. Esta explosión genera una presión de hasta $413 \mathrm{MPa}$, la cual impulsa un pistón comprimiendo un gas de bajo peso molecular, como el helio, hidrógeno o nitrógeno. La compresión de este gas de bajo peso molecular genera presiones cercanas a los $690 \mathrm{MPa}$, la cual es liberada al segundo pistón llamado tubo de lanzamiento (launch tube) donde el proyectil se acelera hasta que abandona el tubo (Con, 2001; Hardage et al., 1964; Mueller et al., 1991).

La liberación del gas de bajo peso molecular al tubo se hace de manera repentina, esto se logra gracias a un diafragma de ruptura ubicado entre la cámara de bombeo y el tubo de lanzamiento. El material utilizado como diafragma varía dependiendo de las velocidades requeridas en la prueba. Para pruebas de hipervelocidad (> $2000 \mathrm{~m} / \mathrm{s}$ ), se utilizan diafragmas de latón con ranuras en el centro para controlar la presión de ruptu- ra; para velocidades menores existen alternativas como películas poliméricas como el poliester y otros polímeros con altas propiedades mecánicas. Sin embargo, un detalle muy importante en este tipo de diseños es la relativa imprecisión en la velocidad de impacto, lo cual limita la determinación del límite balístico de un material con repeticiones puntuales.

El límite balístico de un material se obtiene promediando seis velocidades de impacto, de las cuales tres de ellas no deben perforar el material y tres si, recomendando un intervalo máximo de $60 \mathrm{~m} / \mathrm{s}$ entre la mínima y la máxima velocidad (Donald et al., 2008). Debido al intervalo de velocidad recomendado, es necesario controlar con mucha precisión la velocidad de impacto, lo cual se logra con un cañón de gas ligero de una etapa. Su funcionamiento se basa del TSLGG, con la diferencia de que el gas de bajo peso molecular se comprime con ayuda de un compresor o directamente de los cilindros presurizados comerciales, por ello su velocidad de prueba se limita a velocidades inferiores a $\operatorname{los} 1500 \mathrm{~m} / \mathrm{s}$.

Entre las ventajas que presenta un cañón de gas de una sola etapa, está la versatilidad que estos dispositivos poseen, lo que los hace más precisos en la velocidad de impacto. Otra característica es la simplicidad que estos equipos adquieren en comparación al TSLGG, con lo cual sacrifican velocidad, pero se obtienen equipos más pequeños y precisos.

El objetivo de este trabajo se centra en diseñar, construir y probar un cañón de gas ligero de una sola etapa, el cual utiliza nitrógeno presurizado de cilindros comerciales, presentando una buena relación costo-desempeño. Con este equipo es posible caracterizar muestras, tanto poliméricas como metálicas, ante pruebas de medio y alto impacto. El parámetro a controlar y censar es la velocidad de impacto, con la cual se determina el límite balístico del material probado.

\section{Procedimiento}

En esta sección se describe el diseño de cada elemento y la elección de los sistemas utilizados para satisfacer las consideraciones en la construcción de un cañón de gas de una etapa. Cabe mencionar que estas consideraciones propuestas, presentan un balance entre el máximo desempeño del equipo, cuidando el costo de construcción.

\section{Gas a utilizar}

El primer paso para el armado del sistema fue la elección del gas a utilizar. En equipos con alto desempeño 
se utiliza hidrógeno debido a su bajo peso molecular (2 $\mathrm{g} / \mathrm{mol}$ ); sin embargo, se descartó por ser altamente inflamable. Otros gases que podrían utilizarse son el helio $(8 \mathrm{~g} / \mathrm{mol})$ y el nitrógeno $(28 \mathrm{~g} / \mathrm{mol})$, ya que no corroen el tanque de almacenamiento y presentan bajo peso molecular; sin embargo, presentan un desempeño inferior en comparación al hidrógeno, con lo cual se espera un régimen de velocidad medio a alto impacto tal como se aprecia en la tabla 1 (Goigolea, 2000; Perez, 2008).

En términos económicos, el nitrógeno resulta más atractivo, debido a que el helio cuesta aproximadamente 6 veces más que el nitrógeno, por lo que las pruebas en este trabajo se realizaron con el último. Cabe resaltar que cuando sea necesario alcanzar velocidades elevadas es posible instalar cilindros de helio, con lo cual se espera llegar a velocidades cercanas a los $1000 \mathrm{~m} / \mathrm{s}$.

\section{Sistema de carga y almacenamiento del gas}

El sistema de carga comienza con un cilindro comercial presurizado de gas nitrógeno de $8.5 \mathrm{~m}^{3}$. El gas es transportado al tanque por medio de una manguera Parflex $520 \mathrm{~N}-4$ DN06, a la cual se le insertó un capilar en su interior para controlar el flujo de gas desde el tanque y evitar el uso de un regulador de presión (figura 1c). Posterior a la manguera se encuentra una válvula solenoide normalmente cerrada marca Parker de la serie Skinner 7000, la cual soporta una presión máxima de $20.68 \mathrm{MPa}$. Esta válvula se controla por un sistema de control de presión marca Turck modelo PD600R, el cual tiene una sensibilidad de hasta $0.069 \mathrm{MPa}$ y una presión máxima de control de $60 \mathrm{MPa}$. Además, el sistema cuenta con una válvula de desfogue de emergencia, igual a la válvula de alimentación, que se utiliza para desfogar el tanque en caso de suspensión de la prueba. En la figura $1 \mathrm{~b}$ se presenta un diagrama del sistema armado.

Para controlar todo el sistema se integró un tablero de control el cual, por medidas de seguridad, se ubica a una distancia considerable del cañón de gas fuera del recinto de prueba. Este tablero incluye un interruptor principal y un paro de emergencia, un botón de carga, un botón de disparo y un botón de desfogue de emergencia. A su vez, este tablero muestra en una pantalla la presión del tanque, y en otra pantalla las lecturas tomadas por los cronógrafos al momento del impacto.

\section{Diseño del tanque de almacenamiento}

La presión máxima de operación del equipo se definió en $24.13 \mathrm{MPa}$ (pensado para que un booster pueda elevar la presión máxima del nitrógeno), ya que el gas nitrógeno en cilindros comerciales posee una presión natural de alrededor de $19.30 \mathrm{MPa}$. Con este valor definido, teóricamente es posible alcanzar velocidades de hasta $700 \mathrm{~m} / \mathrm{s}$ (explicado más adelante), con lo cual se alcanzan velocidades de medio a alto impacto (Goicolea, 2000; Seigel, 1965). Con base en la presión de diseño requerido, se obtuvieron las medidas del recipiente, como el espesor de las paredes y las tapas.

Para determinar el espesor de la pared del recipiente se utilizó la ecuación 1, usada para recipientes cilíndricos; considerando una eficiencia recomendada de la unión $(E)$ de 0.90 , una presión de diseño $(P)$ de 27.74 $\mathrm{MPa}, 15 \%$ superior a la presión máxima de operación como factor de seguridad (Megyesy, 1992), un radio interno del contenedor $(R)$ de $5 \mathrm{~cm}$ y un valor del esfuerzo (S) para acero A516 grado 60 de $103.42 \mathrm{MPa}$, recomendado para este material a temperatura ambiente. Con

Tabla 1. Régimen de velocidad en impacto normal

\begin{tabular}{l|c}
\hline \multicolumn{1}{c|}{ Clasificación } & Intervalo de velocidad $(\mathrm{m} / \mathrm{s})$ \\
\hline Baja velocidad & $\mathrm{V}<50$ \\
Velocidad media & $50<\mathrm{V} \leq 500$ \\
Velocidad alta & $500<\mathrm{V}<2000$ \\
Hipervelocidad & $2000<\mathrm{V}$ \\
\hline
\end{tabular}

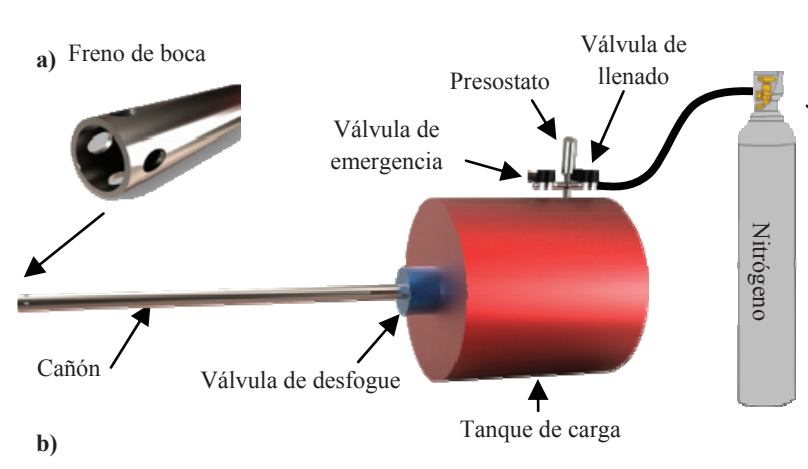

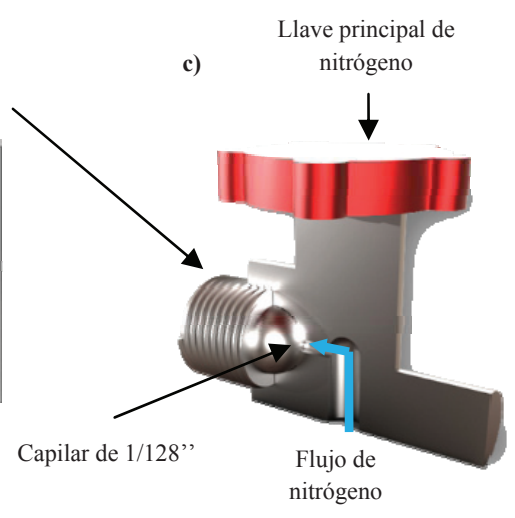

Figura 1. Diagrama del sistema de control de presión de gas, a) freno de boca, b) diagrama del cañón, c) capilar de control de flujo 
estos valores se obtiene el espesor de pared $\left(t_{p}\right)$ mínimo recomendado.

$t_{p}=\frac{P R}{S E-0.6 P}$

Para determinar el espesor de las tapas se utilizó la ecuación 2, esta ecuación se usa en condiciones de presiones internas y externas, para recipientes circulares con tapa plana, diámetros inferiores a $61 \mathrm{~cm}$ y con el espesor de la pared del recipiente inferior al espesor de la tapa. Para el diseño de las tapas se consideró una presión de diseño $(P)$ de $27.74 \mathrm{MPa}$ (con factor de seguridad de $15 \%$ ), un acero A516 grado 60 con un valor del esfuerzo (S) de 103.42 MPa, un valor del espesor mínimo requerido, por presión, del casco sin costura $\left(t_{r}\right)$ de $1.55 \mathrm{~cm}$ y un espesor real del casco $\left(t_{s}\right)$ excluyendo el margen de corrosión de $1.905 \mathrm{~cm}$, ambos valores obtenidos en tablas; y por último una eficiencia recomendada de la junta $(E)$ de 1 . Con estos valores se obtiene el valor de espesor mínimo para las tapas $\left(t_{t}\right)$ frontal y posterior (Megyesy, 1992).

$t_{t}=\sqrt{\frac{0.33 t_{r} P}{t_{s} S E}}$

En la figura 2 se presenta un diagrama del cilindro, así como la entrada y desfogue del mismo con las dimensiones resultantes de los cálculos.

Una consideración importante que involucró el diseño del tanque fue la caída de presión del gas en el cañón. Cuando se carga el tanque se tiene una presión con base en el volumen del mismo (figura 3a); sin embargo, cuando la presión comienza a impulsar el proyectil se tiene una caída de presión debido al cambio del volumen del gas comprimido; donde, idealmente, el volumen máximo que puede alcanzar el gas comprimido sin ser desfogado, es el volumen del tanque más el volumen del cañón (figura 3b). Esta situación se presenta justo un instante antes de que el proyectil abandone el cañón. Para el cálculo de la caída de presión $(\Delta P \%)$ se involucra el volumen del tanque $\left(V_{t}\right)$ cargado a una presión determinada, así como el volumen del cañón $\left(V_{t+c}\right)$, que representa la situación mostrada en la figura 3 b. Con estos valores se puede obtener con la ecuación 3 la caída de presión del gas $\left(\rho_{N}\right)$ en el sistema.

$\Delta P \%=\frac{\left(V_{t}+V_{t+c}\right) \rho_{N} 100 \%}{V_{t}}$

Para este diseño se consideró un volumen de cañón de $1178 \mathrm{~cm}^{3}$ con una caída de presión de alrededor de $6 \%$.
Para valores inferiores a 6\%, se necesita un gran incremento en el volumen del tanque, haciéndolo poco práctico, sin mejorar considerablemente el desempeño del cañón y con mucho desperdicio de gas en cada desfogue, sin alcanzar una ganancia considerable en la velocidad final del proyectil. Este comportamiento se presenta en la figura 4, donde se grafica el cambio del volumen contra la velocidad máxima alcanzada por el equipo. Este comportamiento no lineal está relacionado con el límite de eficiencia que el gas nitrógeno es capaz de alcanzar en este sistema.

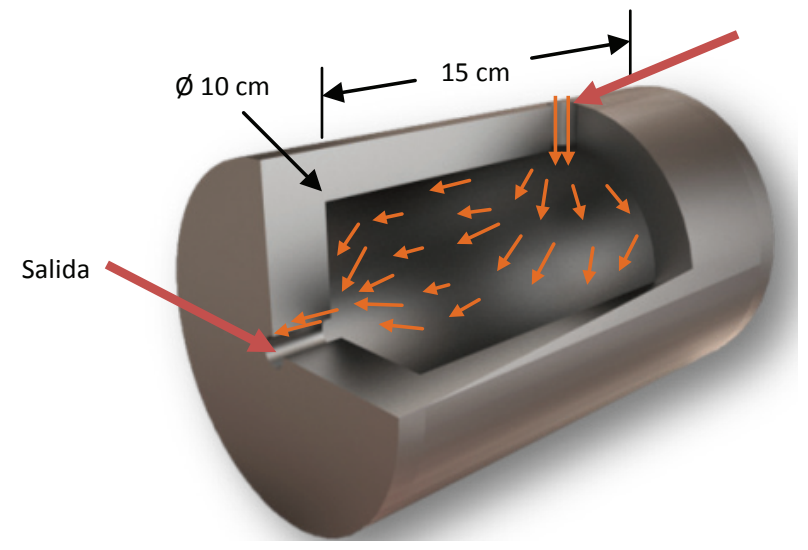

Figura 2. Diagrama del cilindro de gas diseñado para 24.13 MPa

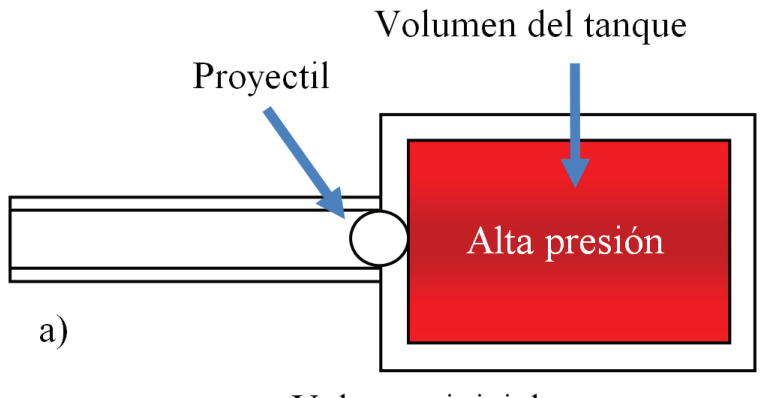

Volumen inicial $=$ tanque

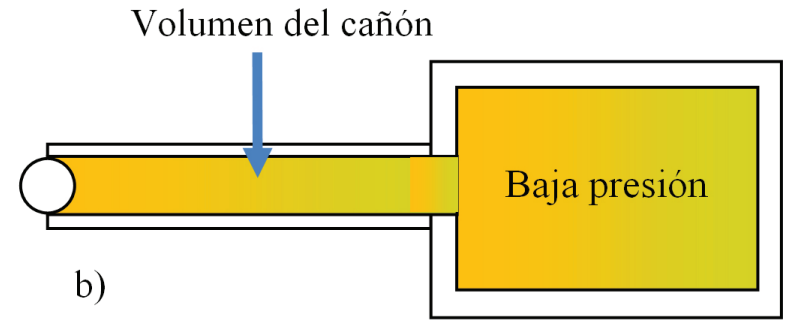

Volumen final $=$ Tanque + cañón

Figura 3. Cambio del volumen del gas comprimido durante un disparo, a) volumen inicial, b) volumen final 


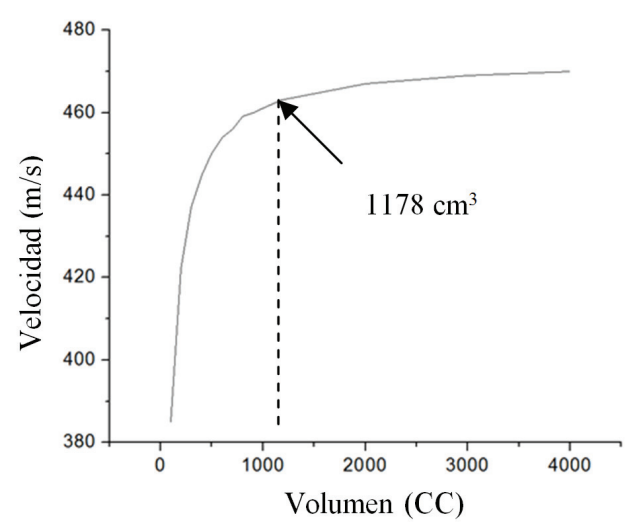

Figura 4. Respuesta de la velocidad con respecto al cambio de volumen del tanque

\section{Sistema de ruptura del diafragma de desfogue instantáneo}

El sistema de desfogue es una parte primordial de un cañón de gas, ya que para generar altas velocidades en el proyectil, se necesita un tiempo de desfogue menor que 0.001 segundos (1 ms). Comercialmente se encuentran modelos de válvulas que alcanzan este tiempo de desfogue; sin embargo, el caudal que alcanzan es muy inferior al que un cañón de gas es capaz de alcanzar en plena aceleración del proyectil, generando una caída de presión importante. Por tal motivo se recurrió a diafragmas poliméricos de ruptura con control térmico. Este sistema funciona por medio de un diafragma, usualmente de poliéster, el cual retiene al gas del tanque de almacenamiento hasta requerirse. Este diafragma está instalado en un accesorio, el cual se calienta por una resistencia eléctrica, donde al calentarse lo suficiente para reblandecer al diafragma, este falla abruptamente rebasado en capacidad por la presión contenida en el tanque. La figura 5 presenta un diagrama de la

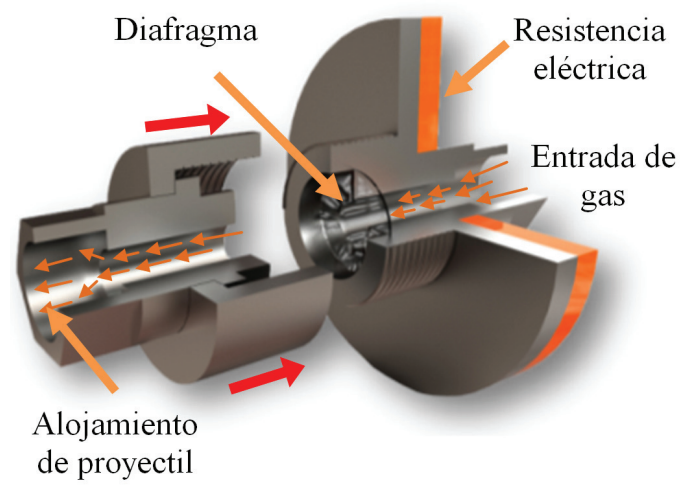

Figura 5. Diagrama de la válvula de desfogue por control térmico válvula diseñada para este propósito, llamada válvula de desfogue por control térmico, mostrando la ubicación del proyectil en la válvula.

Con el fin de obtener un diseño resistente a la presión y la corrosión, la válvula de desfogue por control térmico se fabricó por completo en acero inoxidable, utilizando accesorios también del mismo material. El único inconveniente con este material es su relativa baja conductividad térmica $(14-16 \mathrm{~W} /(\mathrm{m} \times \mathrm{K})$, lo cual prolonga un poco el tiempo de ruptura del material. Sin embargo, al utilizar bronce, que conduce mejor la temperatura, no es posible alcanzar las presiones cercanas a los 20.68 MPa (por su baja resistencia mecánica), es decir, la presión que alcanzan los cilindros comerciales de nitrógeno. La resistencia eléctrica tipo dona instalada en este dispositivo tiene una potencia de 150 Watts, que está en proporción a la velocidad a la que se romperá con éxito el diafragma presurizado para una prueba.

Debido a las presiones utilizadas en este trabajo, se usaron diafragmas de PET y PP, obtenidos de películas comerciales; cada material tiene una presión de ruptura específica, bajo la cual es posible trabajar con precisión con el sistema térmico. Al instalar más de un diafragma en la válvula, es posible duplicar la presión de ruptura, con lo que se tiene la posibilidad de alcanzar elevadas presiones usando un mismo tipo de diafragma.

\section{Diseño y construcción del cañón de disparo}

Otros elementos de gran importancia en el cañón de gas, son precisamente el cañón de disparo y el proyectil, su diseño se basó en la norma STANAG 2920 (Ashok, 2006), que recomienda proyectiles con una masa mínima de $1.02 \mathrm{~g}$. Por esta razón, se utilizó un proyectil comercial esférico con una masa de $1.11 \mathrm{~g}$ de acero al alto vanadio y recubierta de cromo, con un diámetro de $6.75 \mathrm{~mm}$ (Ashok, 2006).

Para otorgar una aceleración eficiente, el proyectil debe viajar por un cañón lo suficientemente ajustado, sin restringir su movimiento. Por ello se eligió un tubo de acero inoxidable con un calibre 18 de pared y $7 \mathrm{~mm}$ de diámetro interior, quedando $0.125 \mathrm{~mm}$ por cada lado entre el proyectil y la pared interior; la presión de servicio que este tubo puede prestar, está reportada en la ficha técnica del material a $25.5 \mathrm{MPa}$, quedando arriba de la presión de diseño del tanque (Swagelok ${ }^{\circledR}, 2014$ ). Es imprescindible que este tubo esté perfectamente alineado, ya que cualquier curvatura podría ocasionar algún desvío en el proyectil al momento del lanzamiento e impacto. Este tipo de cañón simple sin estriado, está 
limitado a proyectiles esféricos que no perturban su dirección fácilmente por su propia forma uniformemente balanceada.

Uno de los parámetros que determinan la eficiencia del equipo es la longitud que el cañón de gas pudiera alcanzar donde, en una condición ideal, el desempeño sería el máximo si la longitud fuera suficientemente larga para que la presión contenida en el tanque caiga hasta la presión atmosférica, cuando el proyectil esté en la boca del cañón y, tanto la fricción como la presión delante del proyectil fuera nula. Esto genera un punto máximo de rendimiento en estos equipos, donde cualquier diseño quedaría por debajo de este parámetro establecido. Esto se debe a que, comercialmente es difícil encontrar cañones con más de 5 metros de longitud. Para este caso se consideró una longitud de 2 metros, generando un desempeño relativamente bajo, pero un equipo lo bastante compacto y funcional para ser alojado en un recinto de tamaño medio.

Para minimizar vibraciones o posibles daños en el sistema por posibles empujes debido al estampido quasi-instantáneo del gas, se maquinaron frenos de boca en la punta del cañón; este genera un pequeño incremento en el ruido generado por un disparo, aunque reduce considerablemente el empuje del disparo generado en el equipo y a su vez, reduce el posible daño causado en la muestra a probar, debido al choque del gas con la misma (figura 1a).

\section{Diseño de la mordaza de sujeción}

Las muestras a probar con el cañón de gas deben estar firmemente sujetas; esto se logra con una mordaza de

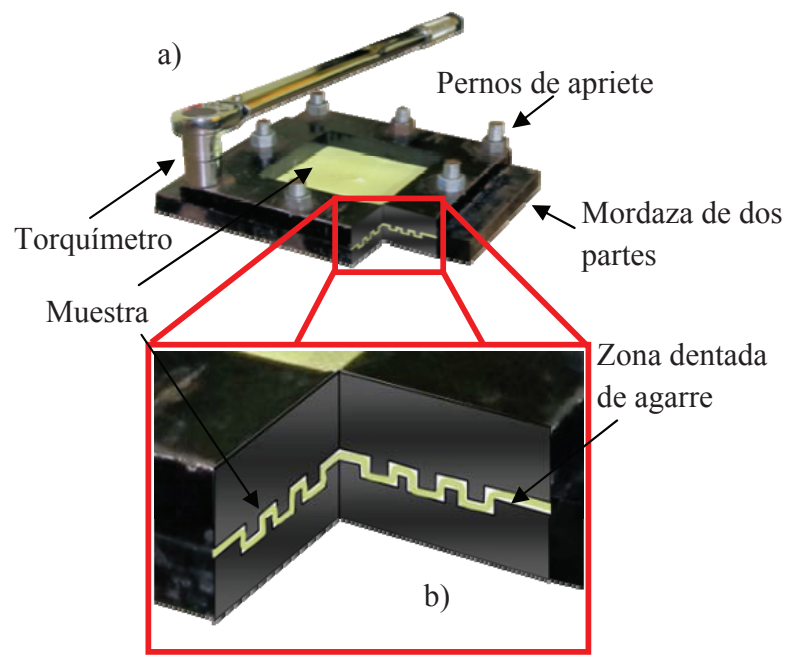

Figura 6. Mordaza de sujeción, a) vista en perspectiva, b) corte transversal de la zona de agarre sujeción de alto agarre. Este dispositivo tiene la capacidad de sujetar eficientemente muestras flexibles y semirrígidas. La capacidad de sujeción se pone a prueba cuando se realizan impactos en muestras flexibles, ya que cualquier deslizamiento entre la muestra y la mordaza representa pérdidas de energía, o sea, puede mostrar un nivel de energía superior al que realmente pudiera estar soportando este material.

Para generar un agarre eficiente en la mordaza diseñada, se generó un dentado entre las caras de sujeción, de tal manera que una muestra flexible sería prácticamente "mordida" por tres dientes ubicados en la mordaza superior, encontrados por tres surcos en la mordaza inferior, con la suficiente precisión para no cortar la muestra a probar. En la figura 6 se ilustra una representación de un corte transversal de la mordaza, donde se observa el dentado y cómo la muestra (en amarillo) copia la forma, garantizando la correcta sujeción del material.

Para obtener una alta precisión en el maquinado de este dispositivo se utilizó una máquina fresadora de control numérico, con lo cual se generó un dentado que coincide adecuadamente con ambas partes del marcomordaza; este punto es muy importante, ya que si no coinciden los dentados (con espacios en donde la muestra se alojaría), podría dar como resultado cortaduras en la muestra. La geometría de la mordaza implementada en este estudio, permite instalar muestras con un área de prueba de $100 \mathrm{~cm}^{2}(10 \mathrm{~cm} \times 10 \mathrm{~cm})$, estas muestras se sujetan firmemente por 8 pernos de $0.95 \mathrm{~cm}$ $\left(3 / 8^{\prime \prime}\right)$ con el apriete uniforme de un torquímetro (mostrado en la figura 6). El espesor de la placa utilizado para las mordazas fue $1.27 \mathrm{~cm}$, con lo que fue posible maquinar surcos de $1 \mathrm{~mm}$ en las caras de sujeción sin comprometer la resistencia de la mordaza.

Por otra parte, esta mordaza se debe sujetar por una base, la cual debe ser suficientemente rígida para evitar vibraciones y posibles fugas de energía. Por esta razón, se construyó una base con perfiles de acero, los cuales están firmemente sujetos a una pared. Con el fin de obtener la máxima versatilidad en el equipo, para esta base se consideró la capacidad de alojar en su interior bloques de gelatina balística o material testigo, los cuales se utilizan ampliamente en estudios de balística terminal (Donald et al., 2008). En la figura 7 se presenta un diagrama de la estructura propuesta.

\section{Sistema de medición de velocidad} y cámara de contención

El sistema de medición de velocidad es una de las partes más importantes de este dispositivo, ya que los re- 
sultados de las pruebas son la velocidad incidente del proyectil sobre la muestra y la velocidad residual (cuando la muestra es penetrada). El dispositivo utilizado para registrar la velocidad del proyectil fue un cronógrafo; en el mercado existen distintos tipos de sistemas para medición de velocidad, que utilizan sistemas infrarrojos u ópticos para esta medición, cada uno con sus ventajas e inconvenientes.

En este equipo se utilizó un cronógrafo de la marca Chrony modelo Alpha Master Chrony, el cual funciona con sensores ópticos y depende de la luz natural (o incandescente) para poder censar algún objeto que pase por su área de registro. Estos cronógrafos están ubicados antes y después de la mordaza de sujeción, y todo esto dentro de un recinto (figura 7a) que contiene los rebotes o material despedido por el impacto. En la figura $7 \mathrm{~b}$ se presenta un diagrama de la cámara de contención, junto con los cronógrafos y la mordaza.

Una consideración que se tomó en cuenta sobre los cronógrafos, es que estos equipos se apoyan en la luz natural para realizar sus mediciones de velocidad, registrando pequeñas variaciones de luz para realizar sus lecturas. Estas variaciones se producen por el proyectil

\section{Alojamiento de gelatina}

balística terminal

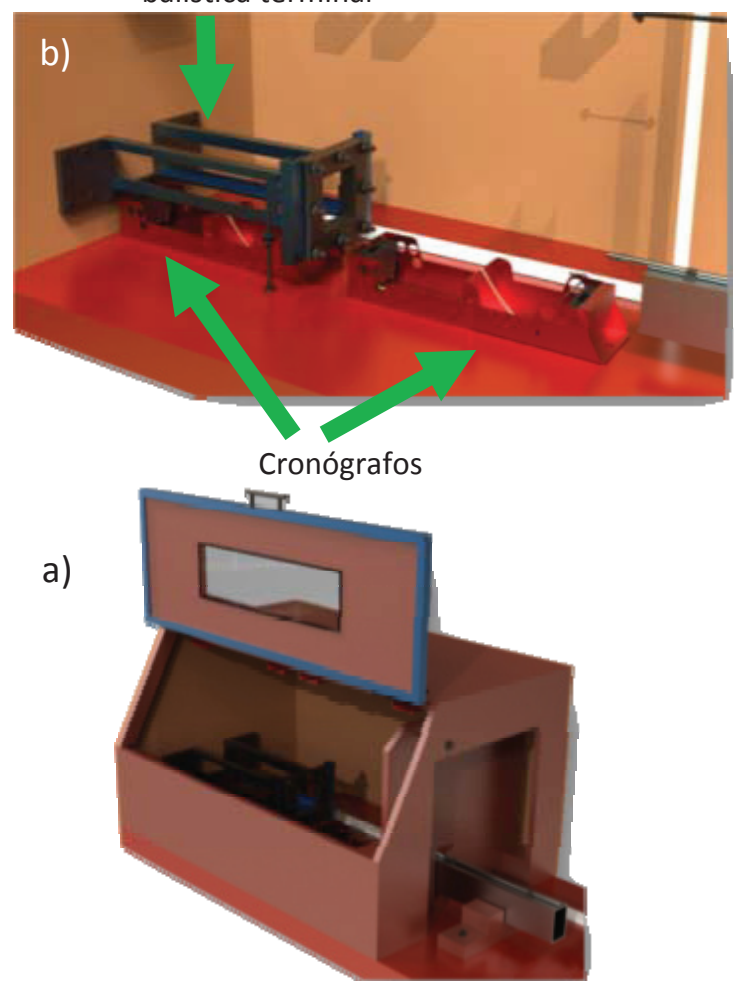

Figura 7. Vista en perspectiva de: a) cámara de contención de impacto, b) ubicación de los cronógrafos que pasa por encima de los sensores generando una interrupción parcial de luz. Esto ocasiona que los cronógrafos no puedan funcionar con luz fluorescente, ya que esta oscila a frecuencias entre 50 y $60 \mathrm{~Hz}$. Por esta razón, fue necesario implementar lámparas incandescentes especiales para este dispositivo; estas lámparas se alojan en el interior de la cámara de impacto encima de cada sensor del cronógrafo.

Para alojar y contener cualquier impacto que pudiera rebotar de la muestra, se diseñó y construyó una cámara de contención de laminado de madera, que contempla pasajes de desfogue para la liberación del gas posterior a un disparo; sin embargo, esta cámara no debe permitir la salida del proyectil por cuestiones de seguridad del usuario. Para este propósito se integraron "trampas", las cuales permiten pasar el gas sin problema y evitar la salida del proyectil desde el interior. Esta cámara tiene una ventana de acceso que cuenta con una pantalla transparente de policarbonato, por donde es posible observar o grabar alguna prueba de impacto realizada en el interior de la cámara.

\section{Cálculo teórico de la velocidad}

Con base en la teoría de diseño balístico, es posible predecir la velocidad máxima que el proyectil en este dispositivo puede alcanzar. La ecuación 4 parte de la ecuación de la ley del gas ideal, lo que hace posible integrar esta ecuación con base en el volumen del gas que se va a utilizar para disparar; parámetro que presenta un dominio importante en la velocidad del proyectil.

$$
W=m_{g} R T_{i} V_{c}^{(\gamma-1)} \int_{V_{c}}^{V} V^{-\gamma} d v
$$

Donde $(W)$ representa el trabajo del gas, suponiendo que toda la energía del gas se convierte en energía cinética considerando pérdidas nulas. Al integrar la ecuación 4 y sustituir el valor de la ecuación de la energía cinética, se obtiene la ecuación 5 (Donald et al., 2008):

$$
\frac{1}{2} m_{p} V^{2}=\frac{m_{g} R T_{i} V_{c}^{(\gamma-1)}}{1-\gamma}\left[V_{F}^{1-\gamma}-V_{c}^{1-\gamma}\right]
$$

Al despejar $(V)$, que representa la velocidad del proyectil, se obtiene la ecuación 6

$V=\sqrt{\frac{2 m_{g} R T_{i} V_{c}^{(\gamma-1)}}{(1-\gamma) m_{p}}\left[V_{F}^{1-\gamma}-V_{c}^{1-\gamma}\right]}$

Esta ecuación involucra la masa del gas utilizado por un disparo $\left(m_{g}\right)$, la constante específica del gas $(R)$, la 
temperatura inicial del gas $\left(T_{i}\right)$, el volumen inicial y el volumen final del gas durante el impacto $\left(V_{c}, V_{F}\right)$, la masa del proyectil $\left(m_{p}\right)$ y la razón de los calores del gas $(\gamma)$.

La ecuación 6 se utiliza para predecir la velocidad del cañón sin considerar el diseño. Por ello, se generó una curva de calibración de velocidad contra presión para este equipo en particular, donde la curva obtenida presenta una precisión mayor en la predicción de la velocidad.

\section{Resultados y discusión}

En la figura 8 se presenta una imagen del cañón ya armado. En la figura 8a se observa el módulo de control del equipo, que controla la presión de carga, se muestran las velocidades de impacto y de salida, botones de control de presión, desfogue de emergencia, botón principal y paro de emergencia. En la figura $8 \mathrm{~b}$ se presenta el tanque con las válvulas de llenado y de control. En la figura 8c se aprecia la cámara de contención, en la cual se aloja la mordaza, los cronógrafos y la base para materiales de prueba de balística terminal.

El gas utilizado para las pruebas demostró funcionar de manera adecuada, con buena eficiencia en comparación con el aire comprimido, aunque menor eficiencia que el helio. Asimismo, el sistema de carga y control de presión presentó un buen desempeño, teniendo una variación en la presión establecida con la presión real de menos de $0.03 \mathrm{MPa}$; esta característica otorga buen control en la velocidad de impacto en cada prueba. En la figura 9 se presenta la curva generada por medio de la ecuación 6 y los valores obtenidos experimentalmente, caracterizado con un proyectil de $1.11 \mathrm{~g}$ de masa.

El sistema de ruptura de diafragma presentó un buen desempeño en el equipo, funcionando de manera eficiente con diafragmas que van desde polietileno, polipropileno y poliéster; materiales con los cuales se cubre un intervalo de velocidades desde $80 \mathrm{~m} / \mathrm{s}$ hasta 750 $\mathrm{m} / \mathrm{s}$. La resistencia instalada de $150 \mathrm{~W}$ calienta la válvula de forma adecuada, reblandeciendo el material en un periodo de 5 a 15 minutos, a lo cual se le suma un tiempo de 5 min en el montaje de la muestra y proyectil, con lo que se obtiene un tiempo total para una prueba de impacto de alrededor de 20 minutos, que pueden variar dependiendo de la potencia de la resistencia de calentamiento.

Como se expuso, el diseño del contenedor generó un recipiente con un volumen total de $1778 \mathrm{~cm}^{3}$, con un espesor mínimo de la pared $\left(t_{p}\right)$ de $1.8 \mathrm{~cm}$ y un espesor mínimo de las tapas $\left(t_{t}\right)$ de $2.72 \mathrm{~cm}$. Estos valores se ob- tuvieron para un acero A516 grado 60, considerando un factor de seguridad de $15 \%$, con lo que se tiene la seguridad adecuada en el sistema aun cuando el valor de la presión de servicio exceda 15\% (27.74 MPa) de la presión de diseño.

La mordaza de sujeción mostró efectividad de agarre con muestras flexibles aquí probadas, al igual que con muestras semirrígidas. Un punto muy importante que cabe señalar es el uso de un torquímetro para el apriete homogéneo de la mordaza, ya que en muestras flexibles es de suma importancia la tensión del material al momento de ser impactadas, esta tensión la genera el dentado de sujeción al momento de apretar los tornillos del marco contra la mordaza (Smith et al., 1958). Debido a esto, es muy importante generar un

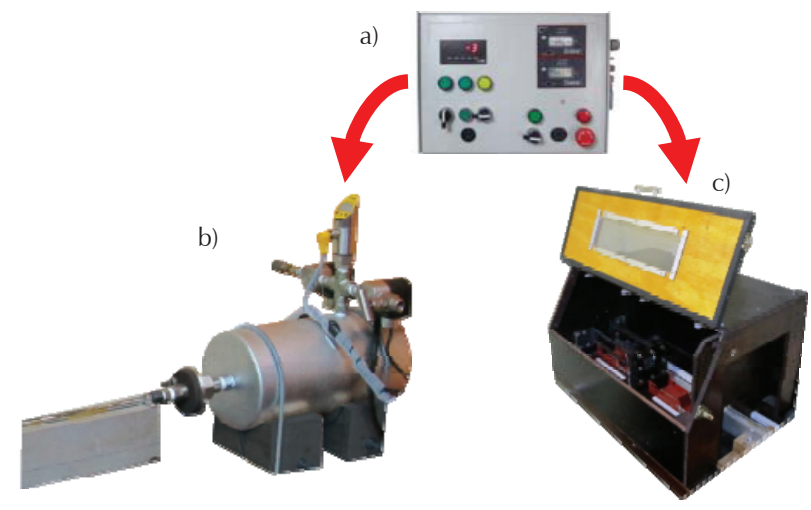

Figura 8. Cañón de gas, a) módulo de control exterior, b) tanque con válvulas de control, c) cámara de contención de impactos

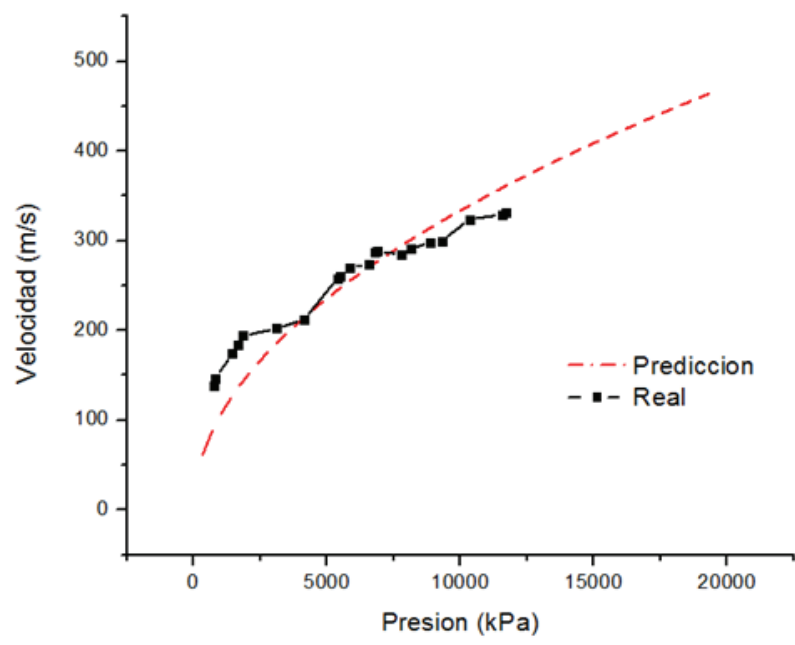

Figura 9. Comparación del comportamiento teórico y experimental de la relación entre la velocidad y la presión de disparo para el presente sistema 


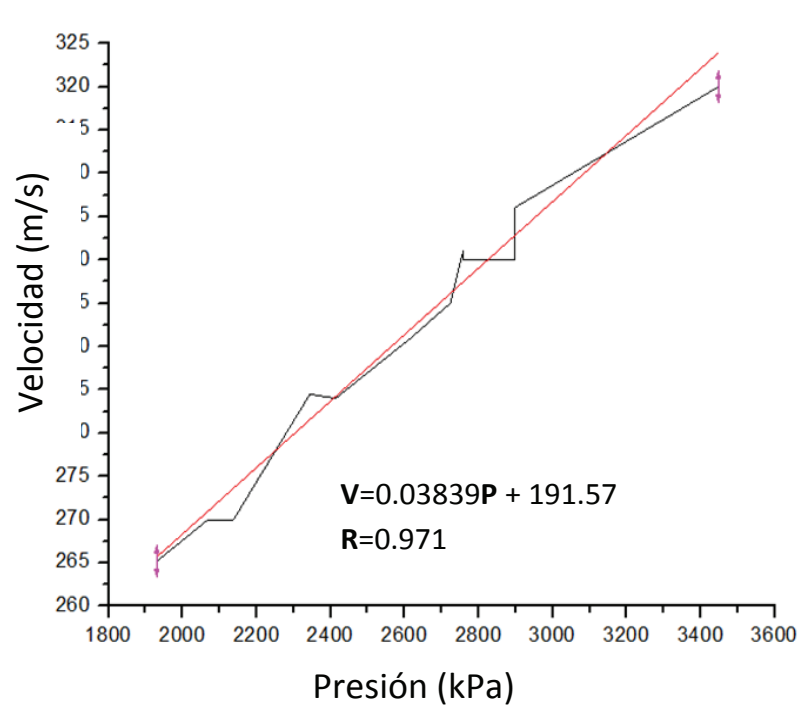

Figura 10. Curva de referencia presión-velocidad para caracterizar el material compuesto Kevlar 720/PP

apriete homogéneo en la muestra, así como similar entre las muestras probadas, para lograr repetibilidad de condiciones. Cabe mencionar que pruebas complementarias con mordaza sin dentado mostraron zonas de deslizamiento después de una prueba de impacto, invalidando la prueba.

Los cronógrafos utilizados para la medición de velocidad han mostrado funcionalidad y precisión de medición de velocidades, aunque en algunas ocasiones no ha sido posible registrar la velocidad residual del proyectil. Esto quizá se debe a las esquirlas que el material probado suelta durante el impacto, las cuales confunden a los sensores del cronógrafo posterior. Por falta de espacio no se pudo alejar el cronógrafo posterior para eliminar este fallo, pero se sigue trabajando buscando tener un equipo más estable.

\section{Validación del equipo}

Para comprobar el funcionamiento del equipo se realizó un estudio para caracterizar un material compuesto de aramida con matriz de polipropileno (PP), con el fin de determinar su límite balístico y sus niveles de absorción de energía en presencia de la matriz de PP y sin ella. El material está constituido por un textil de Kevlar
720 y una matriz de polipropileno atáctico, a una razón de $60 \%$ fracción volumen de fibra (Carrillo et al., 2012).

El procedimiento utilizado para obtener el límite balístico consiste en realizar un impacto a una velocidad estimada a la que se espera el material pudiera no fallar para determinar el límite balístico $\mathrm{V}_{50}$. Si penetra el proyectil, se baja la velocidad de impacto y en caso contrario, se incrementa la velocidad hasta determinar el $V_{50}$.

Se repite este proceso hasta tener seis impactos, de los cuales tres deben penetrar la muestra y tres no; además no se debe tener más de $60 \mathrm{~m} / \mathrm{s}$ de diferencia entre la velocidad más baja y la más alta. Para los impactos que penetraron, es importante registrar la velocidad de salida, ya que con este valor es posible obtener la energía residual del proyectil posterior a un impacto.

Para iniciar las pruebas, es necesario generar una curva de referencia que relacione la velocidad de disparo $(V)$, con la presión generada en el tanque $(P)$; esto se logra haciendo impactos sin muestra en el equipo, empezando con bajas presiones y subiendo a consideración del usuario la presión en cada disparo. Este procedimiento otorga una curva que relaciona la presión alcanzada en el equipo con la velocidad estimada a la cual el proyectil impacta la muestra. En la figura 10 se presenta la curva de referencia y el error $(R)$ obtenidos para estas pruebas.

Ya con la curva determinada, fue posible generar la ecuación de regresión lineal con los puntos obtenidos; con esta ecuación es posible estimar la velocidad que se alcanzará con determinada presión y buena precisión. Este método facilita el uso del equipo y fue posible realizar las pruebas de impacto en el material en cuestión. Se probaron de 1 a 6 capas del material, obteniendo su límite balístico $\left(V_{50}\right)$ y su velocidad de salida $\left(V_{f}\right)$.

En la figura 11 se presentan las imágenes de las pruebas realizadas con estos materiales. En la figura 11a se presenta una muestra de aramida impactada sin perforación, con el fin de medir el trauma (deformación posterior) ocasionado en un material testigo (figura 11b). En la figura 11c se presenta una muestra de arami$\mathrm{da} / \mathrm{PP}$, la cual fue impactada y perforada por el proyectil, el cual se alojó en un bloque de gelatina balística (figura 11d), que ayuda a determinar la energía residual del proyectil. Con esto es posible analizar el comportamiento de un material determinado cuando se perfora o, en caso contrario, cuando no se perfora y presenta un trauma en su parte posterior (Carrillo et al., 2011). 

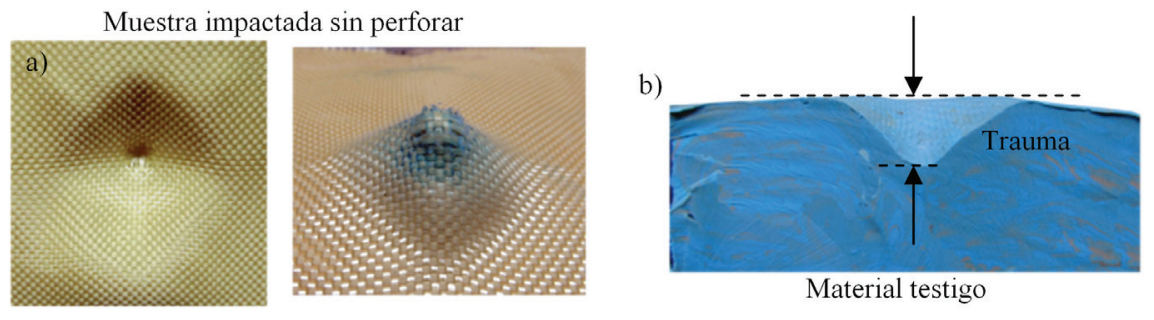

Material testigo

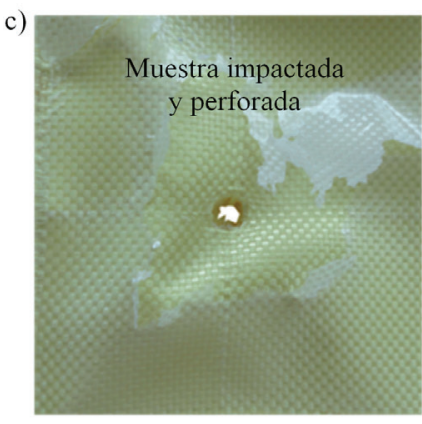

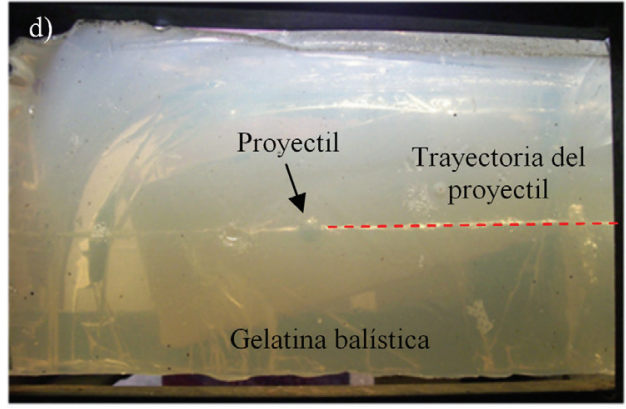

Figura 11. Impactos en: a) muestras de aramida impactadas sin perforar, b) material testigo con marca por deformación de la muestra, c) muestra de aramida perforada, d) gelatina balística con proyectil en su interior

\section{Conclusiones}

Los dispositivos elegidos y construidos en conjunto, otorgaron una efectiva y simple vía para poder construir un cañón de gas para pruebas de impacto. Las pruebas obtenidas indican que pueden alcanzarse velocidades hasta de $750 \mathrm{~m} / \mathrm{s}$, con lo cual se obtiene un intervalo de pruebas de medio impacto e inicios del régimen de alto impacto, presentando bastante precisión en las pruebas realizadas. La predicción de la velocidad realizada en este trabajo, tiene una diferencia de un máximo de $20 \mathrm{~m} / \mathrm{s}$ en comparación con la velocidad obtenida en las pruebas experimentales, con lo cual se tiene una vía confiable para evaluar materiales ante impactos normales para identificar su límite balístico.

Se diseñó y construyó un equipo capaz de realizar pruebas de impacto normal con proyectiles esféricos a velocidades máximas de $700 \mathrm{~m} / \mathrm{s}$, con buena precisión, correspondiente a los datos teóricos obtenidos. Estos parámetros se apegan a estándares internacionales de uso común en balística terminal. Entre las ventajas más importantes que este diseño ofrece está su simplicidad, de fácil construcción e implementación en cualquier ámbito de forma económica. Su versatilidad, otorga la posibilidad de incrementar el desempeño con variables como la longitud del cañón, el tipo de gas o el diámetro interno del cañón, y su potencial en el ámbito de la investigación, permitiendo probar una gran variedad de materiales.

La integración de varios componentes y partes para trabajar en conjunto ofreció una buena compatibilidad en el equipo, con buen desempeño y eficiencia, permitiendo hacer pruebas de impacto en menos de 20 minutos, de inicio a fin. Cabe resaltar que todos los sistemas utilizados son de fácil adquisición, lo cual incluso permite modificar el desempeño del equipo, como al cambiar de tipo de gas, incrementar la longitud del cañón o integrar un compresor de alta presión. Todas estas modificaciones quedan en libertad y posibilidad del diseñador, delimitado por las necesidades particulares de prueba.

Las pruebas realizadas en el equipo demostraron la funcionalidad del sistema, permitiendo encontrar el límite balístico con un promedio de 12 muestras por lote. Con estas pruebas se obtuvo la curva que relaciona el $V_{50}$ del material con el número de capas; siendo de amplia utilidad en calibración de modelos analíticos para validación de nuevos materiales.

\section{Agradecimientos}

Este trabajo se realizó con el apoyo del Consejo Nacional de Ciencia y Tecnología, CONACyT, con el financiamiento del proyecto CB-2008-01-101680.

\section{Referencias}

Ashok Bhatnagar. Lightweight ballistic composite for Military and law-enforcement application, England, CRC Press, 2006.

Carrillo J.G., Gamboa R.A., Flores-Johnson E.A., Gonzalez-Chi P.I. Ballistic performance of thermoplastic composite laminates made from aramid woven fabric and polypropylene matrix. Polymer Testing, volumen 4 (número 31), 2012. 
Carrillo J.G., Gamboa R.A., Cantwell W.J. Advantages of low energy adhesion pp for ballistics, en: Adel Zaki El-Sonbati, Croacia, InTech, 2011, pp. 193-212.

Con D. A two-stage light gas gun for the study of high speed impact in propellants. Defence science \& technology organisation, volumen 20, 2001.

Dirección General de Normas. Niveles de protección de materiales para blindajes resistentes a impactos balísticos-Especificaciones de seguridad y métodos de prueba. México, Diario Oficial de la Federación, vol. 142-SCFI-2000. 2001.

Donald E., Carlucci S., Jacobson S. Ballistics: theory and design of guns and ammunition, Sidney, Australia, CRC Press, 2008.

Goigolea-M. J. Estructuras sometidas a impacto, Madrid, Universidad Politécnica Madrid, 2000.

Hardage B.A., Todd F.C. Design and construction of a helium gal gun for hypervelocity impact. Physical Sciences, volumen 10, 1964.

Megyesy E.F. Manual de recipientes a presión, diseño y cálculo, México, Limusa, 1992.

Mueller A.C., Fernando E.M. The dynamics of projectiles launched by a two-state light-gas gun, Kent, Washington, QUEST Integrated Inc, 1991.

Perez-Campos J.A. Metodología para la simulación de fenómenos de perforacion en placas, debido a fallas del tipo no contenida de turbinas de avión (maestría en tecnología avanzada), México, Insti- tuto Politécnico Nacional, Posgrado en tecnología avanzada, 2008.

Registro Nacional de Armas. Chalecos Antibalas. Ministerio de Justicia, Seguridad y Derechos Humanos, Buenos Aires, volumen 01, 2001.

Seigel E.A. The theory of high speed guns, ADARDograph 91, 1965.

Smith J.C., McCrackin F.L., Schiefer H.F. Stress-strain relationships in yarns subjected to rapid impact loading. Textile Research Journal, volumen 4 (número 28), 1958.

Swagelok ${ }^{\circledR}$, Datos de tubo, www.swagelok.com.mx, 2014.

\section{Este artículo se cita:}

Citación estilo Chicago

Gamboa-Castellanos, Ricardo Alberto, José Gonzálo Carrillo-Baeza, Emmanuel Alejandro Flores-Johnson. Diseño y construcción de un cañon de gas de una etapa para pruebas de impacto de alta velocidad. Ingeniería Investigación y Tecnología, XVI, 02 (2015): 185-195.

\section{Citación estilo ISO 690}

Gamboa-Castellanos R.A., Carrillo-Baeza J.G., Flores-Johnson E.A. Diseño y construcción de un cañon de gas de una etapa para pruebas de impacto de alta velocidad. Ingeniería Investigación y Tecnología, volumen XVI (número 2), abril-mayo 2015: 185-195.

\section{Semblanzas de los autores}

Ricardo Alberto Gamboa-Castellanos. Maestro en ciencias graduado en el Centro de Investigación Científica de Yucatán desde 2011, es asistente de investigador por proyecto del CICY con 17 publicaciones entre artículos arbitrados, artículos de congreso nacionales e internacionales y un capítulo de libro internacional.

José Gonzalo Carrillo-Baeza. Profesor investigador del Centro de Investigación Científica de Yucatán desde el 2008. Es ingeniero mecánico y cuenta con un doctorado en la Universidad de Liverpool, Inglaterra en materiales compuesto avanzados; es miembro continuo del SNI 2008-2015, nivel 1 y cuenta con más de 50 artículos arbitrados y en extenso, internacionales y nacionales publicados, dos patentes en proceso, 30 estudiantes de licenciatura y posgrado graduados como director de tesis. Es docente en el Posgrado de Materiales Poliméricos y Posgrado en Energía Renovable del CICY, con dos proyectos externos vigentes como responsable técnico y dos más como participante.

Emmanuel Alejandro Flores-Johnson. Ingeniero físico graduado de la Universidad Autónoma de Yucatán, con una maestría en materiales poliméricos del Centro de Investigación Científica de Yucatán y un doctorado en ingeniería mecánica de la Universidad de Manchester en Inglaterra. Es miembro del SNI, nivel I, desde el 2012. Actualmente realiza una estancia postdoctoral en la Facultad de Ingeniería Civil de la Universidad de Sydney en Australia. Cuenta con más de 20 publicaciones en revistas internacionales arbitradas y memorias en extenso de conferencias internacionales. Fue co-supervisor de 2 estudiantes de doctorado, 2 de maestría y 5 de licenciatura. 